\title{
The how and why of "negative" research studies
}

\author{
Duminda N. Wijeysundera, MD, PhD • \\ Rita Katznelson, MD
}

Published online: 31 December 2010

(C) Canadian Anesthesiologists' Society 2010

In this issue of the Journal, Bryson et al. ${ }^{1}$ present a singlecentre study that evaluates the inter-relationship between postoperative delirium, postoperative cognitive dysfunction (POCD), and the apolipoprotein E- $\varepsilon 4$ (APOE- $\varepsilon 4$ ) genetic polymorphism. This prospective cohort study, involving 88 individuals who underwent open abdominal aortic aneurysm repair, has many strong points, including strict inclusion criteria, systematic surveillance for delirium and POCD using valid instruments, and clear documentation of postoperative follow-up. The authors found no statistically significant association of delirium with early POCD (odds ratio [OR] 2.86; 95\% confidence interval [CI], 0.99 to 8.27) or with late POCD (OR 2.10; $95 \%$ CI, 0.28 to 15.92 ). They also found no statistically significant association of APOE- $\varepsilon 4$ with delirium (OR 1.63; CI, 0.59 to 4.56 ), early POCD (OR 1.33; CI, 0.44 to 4.04 ), or late POCD (OR 3.64; CI, 0.47 to 28.22). Thus, all of the key study findings were statistically non-significant at the traditional 0.05 level. How then should readers interpret these results? In this article, we present some general approaches both for interpreting these non-statistically significant results and for understanding why the study may have failed to identify associations.

D. N. Wijeysundera, MD, PhD ( $₫)$

Keenan Research Centre, Li Ka Shing Knowledge Institute of St. Michael's Hospital, 80 Bond Street, Toronto, ON M5B 1W8, Canada

e-mail: d.wijeysundera@utoronto.ca

D. N. Wijeysundera, MD, PhD - R. Katznelson, MD

Department of Anesthesia and Pain Management,

Toronto General Hospital, Toronto, ON, Canada

D. N. Wijeysundera, MD, PhD · R. Katznelson, MD Department of Anesthesia, University of Toronto, Toronto, ON, Canada
For the purpose of our discussion, we focus on the estimated association between APOE- $\varepsilon 4$ and postoperative delirium (OR 1.63; CI, 0.59 to 4.56). Rather than interpreting this estimate as showing no association (which the study results do not prove), the authors appropriately interpreted this estimate as showing no statistically significant association between APOE- $\varepsilon 4$ and delirium. However, the key question for most readers is not whether there was a statistically significant association between APOE- $\varepsilon 4$ and delirium but whether there was a clinically significant association.

Man-Son-Hing et al. have proposed an approach for evaluating the clinical significance of study findings. ${ }^{2}$ In this approach, readers must consider both the point estimate (OR 1.63) and the confidence interval (0.59 to 4.56). The CI can be interpreted as the plausible range of values for the true odds ratio within the study population. The CI in this study was quite wide, indicating the imprecision of the study results, likely due to the small sample size. Readers can then define a threshold that they will accept as a clinically meaningful result. For example, if the threshold is defined as an OR of $\geq 3.81$ (the value used in this study for estimating the sample size), the point estimate (1.63) is lower than the threshold, whereas the upper CI (4.56) exceeds the threshold. In this scenario, a clinically significant association is deemed to be "possible". 2 Another approach is to consider primarily the $\mathrm{CI}$ for the $\mathrm{OR}$, i.e., 0.59 to 4.56 . Based on this CI, readers can confidently rule out population odds ratios $<0.6$ and $>4.6$. However, what of all the values in-between? The CI is not "flat", i.e., all of the values within the interval are not equally likely. Values towards the extremes of the interval are less likely than those towards the centre. However, knowing that the values are not equally likely may not be very satisfying to readers 
who want to know the clinical significance, "What is the probability that the OR is X or larger?"

An alternative approach for evaluating the clinical significance of study findings more explicitly is to use Bayesian statistical methods. One of the crucial features of the Bayesian view is that a probability is assigned to a hypothesis (technically, a probability distribution for a parameter of interest, such as the odds ratio); whereas, under the frequentist view expressed previously, a hypothesis is typically rejected or not rejected without directly assigning a probability. To evaluate the probability of a hypothesis, the Bayesian probabilist specifies some prior probability, which is then updated in the light of new relevant data. Although clinicians may initially find this approach unfamiliar, the Bayesian view is used routinely when interpreting diagnostic test results. For example, the estimated risk of a disease (i.e., post-test probability) after diagnostic testing is a function of both the test result and the estimated risk before the test was performed (i.e., pretest probability). Consequently, clinicians appropriately interpret a positive cardiac stress test result differently if the patient is an otherwise healthy 35-yr-old woman with atypical chest pain as opposed to a 50-yr-old male smoker with hypertension. This same approach can be used to interpret research studies, where the new study data (e.g., results of the POISE trial) ${ }^{3}$ are considered in light of the prior probability of the hypothesis being tested (e.g., Do beta blockers prevent perioperative myocardial infarction?). The end result of such an analysis provides a direct answer to the previously stated question, "What is the probability that the OR is X or larger?"

If Bayesian methods can report the results of investigations in a more clinically applicable manner, why then don't researchers commonly report with these methods? Computational difficulties had previously limited the use of these methods; however, these problems have now been largely overcome following the development of computerintensive simulation methods and readily available software. Another concern relates to the use of prior probabilities, which has been criticized as being too "subjective", as opposed to using the seeming "objectivity" of the usual 0.05 threshold for $P$ values in frequentist analyses.

However, it is possible to conduct Bayesian analyses while assuming there is no relevant prior information regarding the treatment effect. Although this assumption is usually not that plausible, this approach produces an estimate that is quantitatively similar to the traditional frequentist CI but still permits reporting of probabilities for a range of clinically relevant effect sizes. ${ }^{4}$ For example, based on this approach, the estimated association between APOE- $\varepsilon 4$ and delirium is consistent with an $82.4 \%$ probability of any increased risk (OR of $\geq 1.0$ ), a $56.3 \%$ probability that the $\mathrm{OR}$ is $\geq 1.5$, a $5.3 \%$ probability that the OR is $>3.81$ (estimate used for sample size calculation), and a $1.6 \%$ probability that the $\mathrm{OR}$ is $\geq 5.0$. A more detailed description of the methods used to calculate these probabilities is provided in our paper on the application of Bayesian methods to interpret randomized trials. ${ }^{4}$

In the remainder of this article, we discuss some plausible reasons why, from a "frequentist" point of view, the estimate was statistically non-significant. First, APOE- $\varepsilon 4$ may simply confer no increased risk of delirium. While possible, it should be noted that a simple Bayesian analysis described above suggests that there is at least an $82 \%$ probability that APOE- $\varepsilon 4$ is associated with some increased risk of delirium. Second, the true association between APOE- $\varepsilon 4$ and delirium may be more moderate in size than assumed by Bryson et al., ${ }^{1}$ thereby rendering their study statistically underpowered. When determining their sample size, the authors assumed that there was a strong association (OR 3.81) between APOE- 84 and delirium. This assumption may have been somewhat optimistic, but it has some precedent. The APOE- $\varepsilon 4$ polymorphism has a very strong association with Alzheimer's dementia (OR 4.1). ${ }^{5}$ Additionally, a previous study in noncardiac surgery found a strong association (OR 3.64) between APOE- $\varepsilon 4$ and delirium, ${ }^{6}$ although aspects of the statistical modelling, namely stepwise variable selection, may have exaggerated this estimate. ${ }^{7}$ Conversely, the true association between APOE- $\varepsilon 4$ and delirium may be an OR of 1.63 or a similarly moderate effect size. It is not unreasonable to expect that a genetic risk factor will, at most, have a moderate effect on the risk of an event with a multifactorial etiology, as is the case with postoperative delirium. For example, genetic markers demonstrate only moderate associations (ORs ranging from 1.08 to 1.38 ) with the development of coronary artery disease. ${ }^{8}$ If the true association between APOE$\varepsilon 4$ and delirium is an OR of 1.63, then Bryson et al.'s study was simply underpowered to detect this more moderatesized association. Based on a $25 \%$ prevalence of APOE- $\varepsilon 4$ in the study sample, 724 participants would have been needed to detect an increase in the risk of delirium from $33 \%$ in non-APOE- $\varepsilon 4$ carriers to $45 \%$ in carriers (two-sided alpha of 0.05 and $80 \%$ power).

Third, there may be a clinically significant true association between APOE- $\varepsilon 4$ and delirium, but aspects of the study design, or bias, attenuated the magnitude of this association. Within the context of this specific study, three general mechanisms may have been responsible for bias. The mechanism most often considered by readers is likely confounding. A confounder is a factor that is associated with both the exposure (i.e., APOE- 84 ) and outcome (i.e., delirium) and may therefore distort their measured relationship. ${ }^{9}$ For example, increased age is a risk factor consistently identified for postoperative delirium. ${ }^{10,11}$ 
Thus, if APOE- $\varepsilon 4$ carriers were systematically younger than non-carriers, the measured association between APOE- 84 and delirium may have been underestimated. Bryson et al. partially addressed this potential for confounding through statistical adjustment by a regression model. After adjusting for a range of risk factors for postoperative delirium, they found minimal differences between the unadjusted and adjusted estimates (OR 1.63; CI, 0.59 to 4.56 vs OR 1.68; CI, 0.60 to 4.66 , respectively). Nonetheless, not all potential confounders were measured and therefore adjusted. For example, readers cannot be certain that imbalances in perioperative benzodiazepine use did not distort the measured association between APOE- $\varepsilon 4$ and delirium. ${ }^{12}$

Aside from confounding, the study estimate could have been influenced by selection bias, where the measured association between APOE- $\_4$ and delirium was distorted by a mechanism through which individuals were selected for participation in the study. For example, APOE- $\varepsilon 4$ may mediate an increased risk for delirium by increasing preexisting cognitive impairment, which is itself a strong risk factor for postoperative delirium. ${ }^{13}$ However, Bryson et al. excluded all individuals with pre-existing dementia from their study; consequently, these exclusion criteria may have reduced the measured association between APOE- $\varepsilon 4$ and delirium. By comparison, a recent cohort study that did not exclude individuals with pre-existing cognitive impairment found a strong association (OR 3.64) between APOE- $\varepsilon 4$ and delirium. ${ }^{6}$ Additionally, APOE- $\varepsilon 4$ may mediate an increased risk for delirium by increasing the burden of atherosclerotic disease ${ }^{14}$ which itself may be a risk factor for postoperative delirium. ${ }^{15}$ However, Bryson et al. ${ }^{1}$ included only individuals undergoing major vascular surgery who invariably have significant atherosclerotic disease whether they are APOE- $\varepsilon 4$ carriers or not. Thus, these inclusion criteria may have diminished the association of APOE- $\varepsilon 4$ with atherosclerotic disease and thereby also its association with delirium. It is possible that APOE$\varepsilon 4$ and delirium have a stronger association among patients undergoing non-vascular procedures, such as major orthopedic surgery.

Finally, differential follow-up of study participants may also bias the estimated association between APOE- $\varepsilon 4$ and delirium. Bryson et al. ${ }^{1}$ did perform excellent and systematic follow-up of study participants using validated instruments for ascertaining delirium and POCD. However, not all participants completed their follow-up assessments. Of the 87 participants who survived their operative procedure (one participant died intraoperatively), three individuals (3.4\%) did not complete their delirium assessment, nine individuals (10.3\%) did not complete their early POCD assessment, and 18 individuals $(20.7 \%)$ did not complete their late POCD assessment. If individuals who did not complete their outcome assessments differed systematically from those who did, there is a potential for biasing the study results. However, the impact of this loss to follow-up can be estimated using a "worst-case" sensitivity analysis. For example, of the three individuals who did not undergo delirium assessments, one was an APOE$\varepsilon 4$ carrier while the others were not. In the most extreme scenario, the missed carrier would have suffered delirium while the non-carriers would not. If these missed individuals had been included in the results, the estimated association between APOE- $\varepsilon 4$ and delirium would have changed only slightly from an OR of 1.63 (CI 0.59 to 4.56) to an OR of 1.98 (CI 0.72 to 5.30).

Having discussed the results of Bryson et al.'s study in detail, what should readers conclude? Is APOE- $\varepsilon 4$ a risk factor for postoperative delirium? As is the case after most studies, the most straightforward answer is that there remains a need for more research. A moderate-sized association between APOE- $\varepsilon 4$ and delirium cannot be ruled out by this study. Additionally, the study inclusion and exclusion criteria may have influenced the magnitude of the estimated effect size. Thus, if a future study is to understand the relationship between APOE- $\varepsilon 4$ and delirium more clearly, it should increase its sample size, include participants with a broader range of preoperative cognitive function, and include more surgical procedures. Should readers be disappointed by the lack of a clear answer from this well-conducted study? No - research and medicine constantly evolve, and Bryson et al. have provided highquality data for better defining how to pursue research into postoperative delirium and POCD.

\section{Le pourquoi et le comment des études de recherche 'négatives'}

Dans ce numéro du Journal, Bryson et coll. ${ }^{1}$ présentent une étude réalisée dans un seul centre qui évalue la relation entre le delirium postopératoire, le dysfonctionnement cognitif postopératoire (DCPO), et le polymorphisme de l'apolipoprotéine E- $\varepsilon 4$ (APOE- $\varepsilon 4$ ). Dans cette étude de cohorte prospective, on a recruté 88 personnes subissant une réparation ouverte d'anévrisme aortique abdominal. L'étude possède de nombreux points forts, notamment des critères d'inclusion rigoureux, une surveillance méthodique du delirium et du DCPO à l'aide d'instruments validés, et une documentation claire du suivi postopératoire. Les auteurs n'ont observé aucune association significative d'un point de vue statistique entre le delirium et un DCPO précoce (rapport de cotes [RC] 2,86; intervalle de confiance [IC] $95 \%, 0,99$ à 8,27) ou un DCPO tardif (RC 2,10; IC $95 \%, 0,28$ à 15,92). Ils n'ont pas trouvé non plus 
d'association significative d'un point de vue statistique entre l'APOE- $\varepsilon 4$ et le delirium ( $\mathrm{RC} 1,63$; IC, 0,59 à 4,56), le DCPO précoce ( $\mathrm{RC} 1,33$; IC, 0,44 à 4,04) ou le DCPO tardif ( $\mathrm{RC} 3,64$; IC, 0,47 à 28,22). En d'autres termes, tous les résultats principaux de l'étude étaient non significatifs d'un point de vue statistique au seuil traditionnel de 0,05. Comment le lecteur devrait-il alors interpréter ces résultats? Dans cet article, nous présentons quelques pistes générales pour nous aider à interpréter ces résultats non significatifs d'un point de vue statistique et comprendre pourquoi l'étude pourrait ne pas avoir pas trouvé d'associations.

Aux fins de notre discussion, nous nous concentrerons sur l'association estimée entre l'APOE- $\varepsilon 4$ et le delirium postopératoire ( $\mathrm{RC} 1,63$; IC, 0,59 à 4,56). Au lieu d'interpréter cette estimation comme ne démontrant pas d'association (ce que les résultats de l'étude ne prouvent pas), les auteurs ont interprété, de façon pertinente, cette estimation comme ne démontrant aucune association significative d'un point de vue statistique entre l'APOE- $\varepsilon 4$ et le delirium. Toutefois, la question importante pour la plupart des lecteurs n'est pas de savoir s'il y avait une association significative d'un point de vue statistique entre l'APOE- 84 et le delirium, mais plutôt de savoir s'il y avait une association significative d'un point de vue clinique.

Man-Son-Hing et coll. ont proposé une approche pour l'évaluation de la signification clinique des résultats d'étude. ${ }^{2}$ Lorsque cette approche est utilisée, le lecteur doit tenir compte autant de l'estimation ponctuelle (RC 1,63) que de l'intervalle de confiance $(0,59$ à 4,56). L'IC peut être interprété comme la fourchette plausible de valeurs du véritable rapport de cotes dans la population à l'étude. Dans cette étude, l'IC était relativement étendu, ce qui indique l'imprécision des résultats de l'étude, probablement due à la taille restreinte de l'échantillon. Le lecteur peut ensuite fixer un seuil qu'il considérera comme donnant un résultat significatif d'un point de vue clinique. Par exemple, si le seuil choisi est déterminé comme un $\mathrm{RC} \geq 3,81$ (la valeur utilisée dans cette étude pour l'estimation de la taille de l'échantillon), alors l'estimation ponctuelle $(1,63)$ est inférieure au seuil, alors que l'IC supérieur $(4,56)$ est plus élevé que le seuil. Dans un tel scénario, une association significative d'un point de vue clinique est jugée 'possible'. ${ }^{2}$ Une autre approche consiste à tenir compte principalement de l'IC pour le RC, soit 0,59 par rapport à 4,56. En se basant sur cet $\mathrm{IC}$, le lecteur peut exclure en toute confiance les rapports de cotes de population $<0,6$ et $>4,6$. Mais qu'en est-il de toutes les valeurs entre les deux? L'IC n'est pas 'une droite' - les valeurs dans l'intervalle ne possèdent pas toutes la même probabilité. Les valeurs proches des extrêmes de l'intervalle sont moins probables que celles situées plus au centre. Toujours est-il que le fait de savoir que les valeurs n'ont pas toutes la même probabilité n'est peut-être pas très satisfaisant pour le lecteur qui veut connaître la signification clinique, soit «Quelle est la probabilité que le $\mathrm{RC}$ soit $\mathrm{X}$ ou plus grand?».

Une autre approche qui peut être utilisée pour évaluer la signification clinique de résultats d'étude de façon plus explicite consiste à utiliser les méthodes statistiques dites bayésiennes. L'une des caractéristiques essentielles de l'approche bayésienne est qu'une probabilité est attribuée à une hypothèse (techniquement parlant, une loi de probabilité pour un paramètre qui nous intéresse, comme le rapport de cotes); alors que dans la vision fréquentiste précédemment décrite, une hypothèse est, en règle générale, rejetée ou non sans attribution directe d'une probabilité. Pour évaluer la probabilité d'une hypothèse, un probabiliste bayésien spécifie une probabilité préalable, laquelle est ensuite ajustée en fonction des nouvelles données pertinentes. Bien que les cliniciens pourraient, de prime abord, trouver cette approche peu familière, la vision bayésienne est fréquemment utilisée dans l'interprétation de résultats de tests diagnostiques. Par exemple, le risque estimé d'une maladie (c.-à-d., la probabilité post-test) après un test diagnostique est fonction du résultat du test et du risque estimé avant que le test ne soit réalisé (c.-à-d. la probabilité pré-test). Par conséquent, les cliniciens interprètent, de façon justifiée, un résultat de test cardiaque positif différemment chez une femme de 35 ans autrement en bonne santé présentant des douleurs thoraciques atypiques et chez un fumeur de 50 ans souffrant d'hypertension. Cette même approche peut être utilisée pour interpréter les études de recherche, où les nouvelles données de l'étude (par ex., les résultats de l'étude POISE) ${ }^{3}$ sont considérées à la lumière de la probabilité préalable de l'hypothèse testée (par ex., les bêtabloquants préviennent-ils l'infarctus du myocarde?). Le résultat final d'une telle analyse offre une réponse directe à la question précédemment posée: «Quelle est la probabilité que le RC soit X ou plus grand?»

$\mathrm{Si}$ les méthodes bayésiennes peuvent rapporter les résultats d'étude d'une manière plus applicable à la clinique, pourquoi les chercheurs ne rapportent-ils pas alors leurs résultats en se servant de ces méthodes? Par le passé, des difficultés au niveau du calcul ont limité l'utilisation de ces méthodes; toutefois, ces problèmes sont maintenant résolus pour la plupart, grâce à la mise au point de méthodes de simulation informatiques à grande capacité de calcul et de logiciels facilement accessibles. Une autre inquiétude est liée à l'utilisation de probabilités préalables, qu'on a critiquée comme étant trop 'subjective', au contraire de l'apparente 'objectivité' du seuil conventionnel de 0,05 pour les valeurs $P$ dans les analyses fréquentistes.

Il est cependant possible de réaliser des analyses bayésiennes tout en présumant qu'il n'y a pas 
d'informations préalables pertinentes quant à l'effet du traitement. Bien que cette supposition ne soit en général pas très plausible, cette approche donne une estimation similaire d'un point de vue quantitatif à l'IC fréquentiste traditionnel, tout en permettant aux auteurs de rapporter les probabilités pour une fourchette de tailles d'effet pertinentes d'un point de vue clinique. ${ }^{4}$ Par exemple, selon cette approche, l'association estimée entre l'APOE- $\varepsilon 4$ et le delirium correspond à une probabilité de $82,4 \%$ de tout risque accru $(\mathrm{RC} \geq 1,0)$, une probabilité de $56,3 \%$ que le $\mathrm{RC}$ soit $\geq 1,5$, une probabilité de $5,3 \%$ que le $\mathrm{RC}$ soit $>$ 3,81 (estimation utilisée pour le calcul de la taille de l'échantillon), et une probabilité de $1,6 \%$ que le RC soit $\geq 5,0$. Nous proposons une description plus détaillée des méthodes utilisées pour calculer ces probabilités dans notre article sur l'application des méthodes bayésiennes pour interpréter les études randomisées. ${ }^{4}$

Dans le reste de cet article, nous présentons certaines raisons plausibles expliquant pourquoi, d'un point de vue 'fréquentiste', l'estimation n'était pas significative d'un point de vue statistique. En premier lieu, il se peut que l'APOE- $\varepsilon 4$ ne confère simplement pas de risque accru de delirium. Bien que cette hypothèse soit possible, il convient de noter qu'une simple approche bayésienne, telle que décrite plus haut, suggère qu'il existe une probabilité d'au moins $82 \%$ que l'APOE- $\varepsilon 4$ soit associée à un risque accru de delirium. Deuxièmement, la véritable association entre l'APOE- $\varepsilon 4$ et le delirium pourrait être de taille plus modérée que la taille présumée par Bryson et coll., ${ }^{1}$ ce qui rendrait leur étude insuffisamment puissante d'un point de vue statistique. Lorsqu'ils ont déterminé la taille d'échantillon, les auteurs ont présumé qu'il existait une association importante (RC 3,81) entre l'APOE- 84 et le delirium. Bien que cette hypothèse ait pu être quelque peu optimiste, elle n'est pas sans précédent. Le polymorphisme de l'APOE- $\varepsilon 4$ entretient une association très forte avec la maladie d'Alzheimer ( $\mathrm{RC} 4,1) .^{5}$ En outre, une étude précédente en chirurgie non cardiaque a démontré qu'il existait une association importante ( $R C$ 3,64) entre l'APOE- $\varepsilon 4$ et le delirium, ${ }^{6}$ bien que certains éléments du modèle statistique, notamment la sélection de variables pas à pas, pourraient avoir gonflé cette estimation. ${ }^{7}$ Inversement, la véritable association entre l'APOE- $\varepsilon 4$ et le delirium pourrait comporter un RC de 1,63 ou une taille d'effet modérée de cette sorte. Il n'est pas déraisonnable de s'attendre à ce qu'un facteur de risque génétique ait, tout au plus, un effet modéré sur le risque d'un événement possédant une étiologie plurifactorielle, comme c'est le cas du delirium postopératoire. Par exemple, les marqueurs génétiques n'ont démontré que des associations modérées ( $\mathrm{RC}$ allant de 1,08 à 1,38) avec le développement de la maladie coronarienne. ${ }^{8} \mathrm{Si}$ la véritable association entre l'APOE- $\varepsilon 4$ et le delirium comporte un $\mathrm{RC}$ de 1,63 , alors l'étude de Bryson et coll. manquait simplement de puissance et n'a pas pu détecter cette association de taille plus modérée. Si l'on se fonde sur la prévalence de $25 \%$ de l'APOE- $\varepsilon 4$ dans l'échantillon de l'étude, il aurait fallu recruter 724 personnes pour détecter une augmentation du risque de delirium de $33 \%$ chez les personnes non porteuses de l'APOE- $\varepsilon 4$ à $45 \%$ chez les porteurs (alpha bilatéral de 0,05 et puissance de $80 \%$ ).

Troisièmement, il pourrait $\mathrm{y}$ avoir une véritable association significative d'un point de vue clinique entre l'APOE- $\varepsilon 4$ et le delirium, mais certains aspects de la méthodologie de l'étude, ou un biais, ont atténué l'ampleur de cette association. Dans le contexte spécifique de cette étude, trois mécanismes généraux peuvent être à l'origine d'un biais. Le mécanisme auquel les lecteurs pensent le plus souvent est probablement le facteur parasite. Un facteur parasite est un facteur associé à l'exposition (soit l'APOE- $\varepsilon 4$ ) et au résultat (soit le delirium) et qui peut dès lors fausser leur relation mesurée. ${ }^{9}$ Par exemple, un âge plus avancé est un facteur de risque couramment identifié de delirium postopératoire. ${ }^{10,11}$ Donc, si les porteurs d'APOE- 84 étaient systématiquement plus jeunes que les non-porteurs, l'association mesurée entre l'APOE- $\varepsilon 4$ et le delirium pourrait avoir été sous-estimée. Bryson et coll. ont partiellement tenu compte de cette variable parasite potentielle en procédant à un ajustement statistique à l'aide d'un modèle de régression. Après avoir ajusté leurs résultats pour tenir compte de plusieurs facteurs de risque de delirium postopératoire, les auteurs ont trouvé des différences minimes entre les estimations ajustées et non ajustées (RC 1,63; IC, 0,59 à 4,56 vs RC 1,68; IC, 0,60 à 4,66, respectivement). Malgré tout, toutes les variables parasites potentielles n'ont pas été mesurées et ajustées en conséquence. Par exemple, le lecteur ne peut pas être certain que les différences dans l'utilisation périopératoire de benzodiazépines n'aient pas biaisé l'association mesurée entre l'APOE- $\varepsilon 4$ et le delirium/ ${ }^{12}$

Outre le biais inhérent aux facteurs parasites, l'estimation de l'étude aurait pu être influencée par un biais de sélection, cas dans lequel l'association mesurée entre l'APOE- $\varepsilon 4$ et le delirium aurait été biaisée par le mécanisme de sélection des participants à l'étude. Par example, l'APOE- $\varepsilon 4$ pourrait servir de médiateur pour augmenter le risque de delirium en exacerbant un trouble cognitif préexistant, ce qui est, en soi, un facteur de risque important de delirium postopératoire. ${ }^{13}$ Toutefois, Bryson et coll. ont exclu de leur étude toutes les personnes souffrant de démence préexistante; par conséquent, ces critères d'exclusion auraient pu réduire l'association mesurée entre l'APOE- $\varepsilon 4$ et le delirium. En comparaison, dans une étude de cohorte récente qui n'a pas exclu les personnes présentant un trouble cognitif préexistant, les auteurs ont observé une association importante (RC 3,64) entre 
l'APOE- $\varepsilon 4$ et le delirium. ${ }^{6}$ De plus, l'APOE- $\varepsilon 4$ pourrait jouer un rôle dans l'augmentation du risque de delirium en augmentant le fardeau de la maladie athéroscléreuse, ${ }^{14}$ laquelle pourrait être un facteur de risque de delirium postopératoire en soi. ${ }^{15}$ Toutefois, Bryson et coll. ${ }^{1}$ n'ont inclus que les personnes devant subir une chirurgie vasculaire majeure, lesquelles souffrent généralement toutes de maladie athéroscléreuse, qu'elles soient porteuses de l'APOE- $\varepsilon 4$ ou non. Dès lors, ces critères d'inclusion pourraient avoir réduit l'association entre l'APOE- $\varepsilon 4$ et la maladie athéroscléreuse et, ainsi, son association au delirium. Il est possible que l'APOE- $\varepsilon 4$ et le delirium entretiennent une association plus forte chez les patients subissant des interventions non vasculaires, comme par exemple une chirurgie orthopédique majeure.

Enfin, le suivi différentiel des participants à l'étude pourrait également biaiser l'association estimée entre l'APOE- $\varepsilon 4$ et le delirium. Bryson et coll. ${ }^{1}$ ont fait un excellent suivi systématique des participants à l'étude à l'aide d'instruments validés afin de déterminer la présence de delirium et de DCPO. Cependant, les participants n'ont pas tous complété leurs évaluations de suivi. Sur les 87 participants qui ont survécu à l'intervention (un participant est décédé pendant l'opération), trois personnes $(3,4 \%)$ n'ont pas complété leur évaluation du delirium, neuf personnes $(10,3 \%)$ n'ont pas complété leur évaluation de DCPO précoce, et 18 personnes $(20,7 \%)$ n'ont pas complété leur évaluation de DCPO tardif. Si les personnes n'ayant pas complété les évaluations de suivi étaient systématiquement différentes de celles qui les ont complété, cela pourrait biaiser les résultats de l'étude. Toutefois, on peut estimer l'impact de ces personnes perdues au suivi à l'aide d'une analyse de sensibilité au «pire cas». Par exemple, parmi les trois personnes qui n'ont pas fait d'évaluation de delirium, l'une était porteuse de l'APOE- $\varepsilon 4$ mais pas les deux autres. Dans le scénario le plus extrême, la personne porteuse non comptabilisée aurait souffert de delirium, mais pas les non-porteuses. Si ces personnes manquant aux résultats y avaient été incluses, l'association estimée entre l'APOE- $\varepsilon 4$ et le delirium n'aurait changé que légèrement, passant d'un RC de 1,63 (IC 0,59 à 4,56) à un RC de 1,98 (IC 0,72 à 5,30).

Après cette discussion détaillée des résultats de l'étude de Bryson et coll., que devrait conclure le lecteur? L'APOE- $\varepsilon 4$ est-elle un facteur de risque de delirium postopératoire? Tout comme c'est le cas après la plupart des études, la réponse la plus simple est qu'il faut effectuer davantage de recherches. Cette étude ne permet pas d'exclure une association de taille modérée entre l'APOE- $\varepsilon 4$ et le delirium. En outre, les critères d'inclusion et d'exclusion de l'étude ont pu influencer la taille estimée de l'effet. Dès lors, si une étude souhaite à l'avenir mieux comprendre la relation entre l'APOE- $\varepsilon 4$ et le delirium, elle devra augmenter sa taille d'échantillon, inclure des participants présentant une gamme plus étendue de fonctions cognitives préopératoires, et inclure davantage d'interventions chirurgicales. Le lecteur devrait-il être déçu de l'absence de réponse claire de cette excellente étude? Non - la recherche et la médecine sont en constante évolution, et Bryson et coll. nous ont offert des données d'excellente qualité qui nous permettront de mieux définir la manière dont il faut poursuivre nos recherches pour comprendre le delirium postopératoire et le DCPO.

Acknowledgements Dr. Wijeysundera is supported by a ClinicianScientist Award from the Canadian Institutes of Health Research. Drs. Wijeysundera and Katznelson are supported by Merit Awards from the Department of Anesthesia at the University of Toronto.

Competing interests None declared.

\section{References}

1. Bryson GL, Wyand A, Wozny D, Rees L, Taljaard M, Nathan H. A prospective cohort study evaluating associations among delirium, postoperative cognitive dysfunction, and apolipoprotein E genotype following open aortic repair. Can J Anesth 2011; 58. DOI: 10.1007/s12630-010-9446-6.

2. Man-Son-Hing M, Laupacis A, O'Rourke K, et al. Determination of the clinical importance of study results. J Gen Intern Med 2002; 17: 469-76.

3. Devereaux PJ, Yang H, Yusuf S, et al.; POISE Study Group. Effects of extended-release metoprolol succinate in patients undergoing non-cardiac surgery (POISE trial): a randomised controlled trial. Lancet 2008; 371: 1839-47.

4. Wijeysundera DN, Austin PC, Hux JE, Beattie WS, Laupacis A. Bayesian statistical inference enhances the interpretation of contemporary randomized controlled trials. J Clin Epidemiol 2009; 62: 13-21.e5.

5. Bertram L, McQueen MB, Mullin K, Blacker D, Tanzi RE. Systematic meta-analyses of Alzheimer disease genetic association studies: the AlzGene database. Nat Genet 2007; 39: 17-23.

6. Leung JM, Sands LP, Wang Y, et al. Apolipoprotein E e4 allele increases the risk of early postoperative delirium in older patients undergoing noncardiac surgery. Anesthesiology 2007; 107: 406-11.

7. Steyerberg EW. Clinical Prediction Models: A Practical Approach to Development, Validation, and Updating. New York, NY: Springer; 2009.

8. Samani NJ, Erdmann J, Hall AS, et al. Genomewide association analysis of coronary artery disease. N Engl J Med 2007; 357: 443-53.

9. Savitz DA. Interpreting Epidemiologic Evidence: Strategies for Study Design and Analysis. New York, NY: Oxford University Press; 2003.

10. Katznelson R, Djaiani G, Mitsakakis $N$, et al. Delirium following vascular surgery: increased incidence with preoperative betablocker administration. Can J Anesth 2009; 56: 793-801.

11. Katznelson R, Djaiani GN, Borger MA, et al. Preoperative use of statins is associated with reduced early delirium rates after cardiac surgery. Anesthesiology 2009; 110: 67-73.

12. Marcantonio ER, Juarez G, Goldman L, et al. The relationship of postoperative delirium with psychoactive medications. JAMA 1994; 272: 1518-22. 
13. Robinson TN, Raeburn CD, Tran ZV, Angles EM, Brenner LA, Moss $M$. Postoperative delirium in the elderly: risk factors and outcomes. Ann Surg 2009; 249: 173-8.

14. Lahoz C, Schaefer EJ, Cupples LA, et al. Apolipoprotein E genotype and cardiovascular disease in the Framingham Heart Study. Atherosclerosis 2001; 154: 529-37.
15. Rudolph JL, Babikian VL, Birjiniuk $V$, et al. Atherosclerosis is associated with delirium after coronary artery bypass graft surgery. J Am Geriatr Soc 2005; 53: 462-6. 